PROCEEDINGS OF THE

AMERICAN MATHEMATICAL SOCIETY

Volume 137, Number 8, August 2009, Pages 2541-2555

S 0002-9939(09)09800-1

Article electronically published on January 28, 2009

\title{
THE SCATTERING MATRIX FOR THE HILBERT MODULAR GROUP
}

\author{
RIAD MASRI
}

(Communicated by Ken Ono)

\begin{abstract}
In this paper, we compute the scattering matrix for the Hilbert modular group over any number field $K$. We then compute the determinant of the scattering matrix and show it is a ratio of completed Dedekind zeta functions associated to the Hilbert class field of $K$. This generalizes work of Efrat and Sarnak in the imaginary quadratic case.
\end{abstract}

\section{InTRODUCTION AND STATEMENTS OF RESULTS}

Let $X$ be a non-compact, finite volume quotient of a rank one symmetric space $G / K$ by a discrete subgroup $\Gamma<G$. The eigenvalues $\lambda$ corresponding to cuspidal eigenfunctions of the Laplacian $\Delta$ in $L^{2}(X)$ form a discrete set. Let $\mathrm{N}(T)$ count the number of eigenvalues $\lambda \leq T$. In order to prove Weyl's law for $\mathrm{N}(T)$, one must estimate the contribution of the continuous spectrum in the Selberg trace formula, which involves the logarithmic derivative of the determinant of a scattering matrix $\Phi(s)$ (see [Sa]). In particular, if one can prove that $\phi(s)=\operatorname{det}(\Phi(s))$ is a ratio of entire functions of order one, standard methods from analytic number theory can be used to deduce Weyl's law. While this can be accomplished using representationtheoretic methods (see $[\mathbb{R}]$ ), it is often useful to have an explicit formula for $\phi(s)$, and for this purpose classical methods work best.

In this paper, we compute the scattering matrix and determinant for the Hilbert modular group over any number field. In order to state our results, we fix the following notation. Let $K$ be a number field of degree $N=r_{1}+2 r_{2}$ over $\mathbb{Q}$. Let $\mathcal{Q}_{K}^{2}$ be the symmetric space of positive 2 -forms $Q$ over $K$, which consists of $\left(r_{1}+r_{2}\right)$-tuples $Q=\left(Q^{(1)}, \ldots, Q^{\left(r_{1}+r_{2}\right)}\right)$ of positive definite $2 \times 2$ matrices such that the first $r_{1}$ are real symmetric and the second $r_{2}$ are complex Hermitian. Define the quotient $X=\mathrm{SL}_{2}\left(\mathcal{O}_{K}\right) \backslash \mathcal{S} \mathcal{Q}_{K}^{2}$, where $\mathcal{S} \mathcal{Q}_{K}^{2}$ is the determinant one subspace of $\mathcal{Q}_{K}^{2}$. It is well known that there is a bijection between the cusp classes $\left[x_{i}\right]$ of $X$ and the ideal classes $\left[\mathfrak{a}_{i}\right]$ of $K$. Let $\operatorname{cl}\left(\mathcal{O}_{K}\right)$ be the ideal class group and $h=\# \operatorname{cl}\left(\mathcal{O}_{K}\right)$ be the class number of $K$. There is an Eisenstein series $E_{\left[x_{i}\right]}(Q, s)$ corresponding to each cusp class $\left[x_{i}\right]$ which satisfies a functional equation under the involution $(Q, s) \mapsto\left(Q^{-1}, 1-s\right)$. Therefore, the vector Eisenstein series

$$
\overrightarrow{\mathbf{E}}(Q, s)=\left(E_{\left[x_{1}\right]}(Q, s), \ldots, E_{\left[x_{h}\right]}(Q, s)\right)^{t}
$$

Received by the editors July 9, 2008, and, in revised form, October 13, 2008.

2000 Mathematics Subject Classification. Primary 11F41.

(C)2009 American Mathematical Society 
satisfies a functional equation of the form

$$
\overrightarrow{\mathbf{E}}(Q, s)=\Phi(s) \cdot \overrightarrow{\mathbf{E}}\left(Q^{-1}, 1-s\right)
$$

for some $h \times h$ matrix $\Phi(s)$. The scattering matrix is defined to be $\Phi(s)$, and the scattering determinant is defined to be $\phi(s)=\operatorname{det}(\Phi(s))$.

Let

$$
G(s)=\left(2^{-r_{2}} d_{K}^{\frac{1}{2}} \pi^{-\frac{N}{2}}\right)^{s} \Gamma\left(\frac{s}{2}\right)^{r_{1}} \Gamma(s)^{r_{2}},
$$

where $d_{K}$ is the absolute value of the discriminant of $K$. Let

$$
\|Q\|=\prod_{j=1}^{N}\left|\operatorname{det}\left(Q^{(j)}\right)\right|
$$

be the norm of the determinant of $Q$, where $Q^{\left(r_{1}+r_{2}+j\right)}:=\overline{Q^{\left(r_{1}+j\right)}}$ for $j=1, \ldots, r_{2}$, and define the $h \times h$ diagonal matrix

$$
\mathbf{D}(Q)=\left(\begin{array}{lll}
\|Q\| & & \\
& \ddots & \\
& & \|Q\|
\end{array}\right) .
$$

Let

$$
\zeta_{\left[\mathfrak{a}_{i}^{-1} \mathfrak{a}_{j}\right]}(s)=\sum_{(0) \neq \mathfrak{b} \in\left[\mathfrak{a}_{i}^{-1} \mathfrak{a}_{j}\right]} N_{K / \mathbb{Q}}(\mathfrak{b})^{-s}, \quad \operatorname{Re}(s)>1,
$$

be the $(i, j)$-th entry of the $h \times h$ matrix $\left(\zeta_{\left[\mathfrak{a}_{i}^{-1} \mathfrak{a}_{j}\right]}(s)\right)$ of ideal class Dedekind zeta functions. Finally, let $\mathbf{P}$ be the $h \times h$ permutation matrix corresponding to the involution of $\operatorname{cl}\left(\mathcal{O}_{K}\right)$ given by $\mathfrak{a} \rightarrow \mathfrak{a}^{*}$.

In the following theorem, we give an explicit formula for the scattering matrix $\Phi(s)$ in the functional equation satisfied by $\overrightarrow{\mathbf{E}}(Q, s)$.

Theorem 1.1. The vector Eisenstein series $\overrightarrow{\mathbf{E}}(Q, s)$ satisfies the functional equation

$$
\overrightarrow{\mathbf{E}}(Q, s)=\Phi(s) \cdot \overrightarrow{\mathbf{E}}\left(Q^{-1}, 1-s\right),
$$

where the $h \times h$ scattering matrix $\Phi(s)$ is given by

$$
\Phi(s)=\frac{G(2(1-s))}{G(2 s)} \sqrt{\mathbf{D}\left(Q^{-1}\right)} \cdot\left(\zeta_{\left[\mathfrak{a}_{i}^{-1} \mathfrak{a}_{j}\right]}(2 s)\right)^{-1} \cdot \mathbf{P} \cdot\left(\zeta_{\left[\mathfrak{a}_{i}^{-1} \mathfrak{a}_{j}\right]}(2(1-s))\right) .
$$

Using that $\mathbf{P}^{2}=\mathbf{I}_{h}$, we obtain from Theorem 1.1 the following functional equation for $\Phi(s)$.

Corollary 1.2. The scattering matrix $\Phi(s)$ satisfies the functional equation

$$
\Phi(s) \Phi(1-s)=\mathbf{D}\left(Q^{-1}\right) .
$$

In the following theorem, we give an explicit formula for the scattering determinant $\phi(s)=\operatorname{det}(\Phi(s))$.

Theorem 1.3. Let $m$ be the order of the $\operatorname{group} \operatorname{cl}\left(\mathcal{O}_{K}\right)[2]$ of 2-torsion in $\operatorname{cl}\left(\mathcal{O}_{K}\right)$. Then

$$
\phi(s)=(-1)^{\frac{h-m}{2}}\left\|Q^{-1}\right\|^{\frac{h}{2}} \frac{\xi_{H}(2(1-s))}{\xi_{H}(2 s)},
$$


where $\xi_{H}(s)=G(s)^{h} \zeta_{H}(s)$ is the completed Dedekind zeta function of the Hilbert class field $H$ of $K$.

Remark 1.4. In [ES], Efrat and Sarnak computed the scattering matrix and determinant for $\operatorname{PSL}_{2}\left(\mathcal{O}_{D}\right) \backslash \mathbb{H}^{3}$, where $\mathbb{H}^{3}$ is hyperbolic three-space and $\operatorname{PSL}_{2}\left(\mathcal{O}_{D}\right)$ is the Bianchi group corresponding to the imaginary quadratic field $K=\mathbb{Q}(\sqrt{-D})$, $D \neq 1,3$. Theorem 1.3 generalizes [ES, Theorem 1] to arbitrary number fields. Note that the Eisenstein series $\overrightarrow{\mathbf{E}}(w, s)$ studied in [ES] is scaled so that the critical line is $\operatorname{Re}(s)=1$ and normalized so that the fundamental domain has volume one. In Proposition 2.7, we give the precise relationship between $\overrightarrow{\mathbf{E}}(Q, s)$ and $\overrightarrow{\mathbf{E}}(w, s)$.

Remark 1.5. It can be shown using genus theory that if $K$ is imaginary quadratic, then $m=2^{t-1}$, where $t$ is the number of prime divisors of $d_{K}$ (see [H] Theorem 132]).

Finally, in the following theorem we give an explicit formula for the trace of $\Phi(s)$ at $s=\frac{1}{2}$.

Theorem 1.6. With notation as above,

$$
\operatorname{Tr}\left(\Phi\left(\frac{1}{2}\right)\right)=\left\|Q^{-1}\right\|^{\frac{1}{2}}(m-2) .
$$

Remark 1.7. Theorem 1.6 has the following application. Suppose that $K$ is imaginary quadratic and $Q$ is in $\mathcal{S} \mathcal{Q}_{K}^{2}$. Let $\beta:=\sqrt{2} / d_{K}^{\frac{1}{4}}$ and define the normalized Eisenstein series

$$
\widetilde{E}_{\left[x_{i}\right]}(Q, s):=2 \beta^{s} E_{\left[x_{i}\right]}(Q, s / 2) .
$$

The dimension of the vector space $V=\operatorname{span}\left\{\widetilde{E}_{\left[x_{i}\right]}(Q, 1): i=1, \ldots, h\right\}$ appears in the Selberg trace formula and the Lax-Phillips-Sarnak scattering theory for automorphic functions (see $[\mathrm{LP}, \overline{\mathrm{PS}}]$ ). Using properties of the scattering matrix for the normalized vector Eisenstein series, one can show that

$$
\operatorname{dim}(V)=\frac{h+\operatorname{Tr}\left(\Phi\left(\frac{1}{2}\right)\right)}{2} .
$$

It follows from Theorem 1.6 with $\left\|Q^{-1}\right\|=1$ that $\operatorname{dim}(V)=\frac{h+m}{2}-1$.

To compute the scattering matrix, one typically uses the Fourier expansions of the Eisenstein series in the various cusps. In work closely related to ours, Sorensen So. computed the scattering matrix for $\mathrm{SL}_{2}\left(\mathcal{O}_{K}\right) \backslash\left(\mathbb{H}^{2}\right)^{r_{1}} \times\left(\mathbb{H}^{3}\right)^{r_{2}}$ where $K$ is any number field by defining the analog of the Eisenstein series $\overrightarrow{\mathbf{E}}(w, s)$ for this quotient and using the Fourier expansions of the Eisenstein series in the various cusps. In contrast, to compute $\Phi(s)$ we relate $\overrightarrow{\mathbf{E}}(Q, s)$ to a vector Epstein zeta function

$$
\overrightarrow{\mathbf{Z}}_{2}(Q, s)=\left(Z_{2}\left(Q,\left[\mathfrak{a}_{1}\right], s\right), \ldots, Z_{2}\left(Q,\left[\mathfrak{a}_{h}\right], s\right)\right)^{t},
$$

and then establish a functional equation for $\overrightarrow{\mathbf{Z}}_{2}(Q, s)$. This is very similar to the approach taken by Efrat and Sarnak in [ES].

\section{SL(2)-EISENSTEIN SERIES}

In this section we define the Eisenstein series to be studied in this paper (see also [Si, T]). 
Definition 2.1. Let $n$ be a positive integer. The symmetric space $\mathcal{Q}_{K}^{n}$ of positive $n$-forms over $K$ consists of $\left(r_{1}+r_{2}\right)$-tuples $Q=\left(Q^{(1)}, \ldots, Q^{\left(r_{1}+r_{2}\right)}\right)$ of positive definite $n \times n$ matrices such that the first $r_{1}$ are real symmetric and the second $r_{2}$ are complex Hermitian. The determinant one subspace $\mathcal{S} \mathcal{Q}_{K}^{n}$ consists of $Q$ in $\mathcal{Q}_{K}^{n}$ such that $\operatorname{det}\left(Q^{(j)}\right)=1$ for $j=1, \ldots, r_{1}+r_{2}$.

The group $\mathrm{GL}_{n}(K)$ acts on $\mathcal{Q}_{K}^{n}$ by

$$
Q[A]=\left({\overline{A^{(1)}}}^{t} Q^{(1)} A^{(1)}, \ldots,{\overline{A^{\left(r_{1}+r_{2}\right)}}}^{t} Q^{\left(r_{1}+r_{2}\right)} A^{\left(r_{1}+r_{2}\right)}\right), \quad A \in \mathrm{GL}_{n}(K),
$$

where $A^{(j)}$ means that the $j$-th embedding of $K$ is applied to each entry of $A$.

The projective line $\mathbb{P}^{1}(K)=K \cup\{\infty\}$ consists of the cusps for the fundamental domain of the quotient $\mathrm{SL}_{2}\left(\mathcal{O}_{K}\right) \backslash \mathcal{S} \mathcal{Q}_{K}^{2}$. The group $\mathrm{SL}_{2}\left(\mathcal{O}_{K}\right)$ acts on $\mathbb{P}^{1}(K)$ by linear fractional transformations, and this induces an equivalence relation on $\mathbb{P}^{1}(K)$. The proof of the following fact can be found in $\mathrm{Si}$.

Proposition 2.2. The action of $\mathrm{SL}_{2}\left(\mathcal{O}_{K}\right)$ on $\mathbb{P}^{1}(K)$ divides $\mathbb{P}^{1}(K)$ into $h$ cusp classes.

Let $\left\{x_{1}, \ldots, x_{h}\right\} \leftrightarrow\left\{\mathfrak{a}_{1}, \ldots, \mathfrak{a}_{h}\right\}$ denote the cusp, ideal class correspondence. Write $x_{i}=p_{i} / s_{i}$ for some $p_{i}, s_{i} \in \mathcal{O}_{K}$, and assume without loss of generality that $x_{1}=\infty=1 / 0$. In the proof of Proposition 2.2, the cusp $x_{i}$ corresponds to the integral ideal $\mathfrak{a}_{i}=\left(p_{i}, s_{i}\right)$. One can choose (not necessarily uniquely) $\tilde{p}_{i}, \tilde{s}_{i} \in \mathfrak{a}_{i}^{-1}$ such that $p_{i} \tilde{s}_{i}-s_{i} \tilde{p}_{i}=1$. Form the corresponding the matrix

$$
A_{i}=\left(\begin{array}{cc}
p_{i} & \tilde{p}_{i} \\
s_{i} & \tilde{s}_{i}
\end{array}\right) \in \mathrm{SL}_{2}(K)
$$

Let $U_{K}$ be the group of units in $K$. The proof of the following fact can also be found in $\mathrm{Si}$.

Proposition 2.3. The stabilizer of the cusp $x_{i}$ can be expressed as

$$
\Gamma_{x_{i}}=\left\{A_{i}\left(\begin{array}{cc}
u & z \\
0 & u^{-1}
\end{array}\right) A_{i}^{-1}: z \in \mathfrak{a}_{i}^{-2}, u \in U_{K}\right\} .
$$

Each $Q$ in $\mathcal{Q}_{K}^{2}$ has a unique vector Iwasawa decomposition whose $j$-th component is given by

$$
Q^{(j)}=\left(\begin{array}{cc}
1 & 0 \\
q^{(j)} & 1
\end{array}\right)\left(\begin{array}{cc}
v^{(j)} & 0 \\
0 & w^{(j)}
\end{array}\right)\left(\begin{array}{cc}
1 & q^{(j)} \\
0 & 1
\end{array}\right),
$$

where $v^{(j)}, w^{(j)}>0$ for $j=1, \ldots, r_{1}+r_{2}$, and

$$
q^{(j)} \in V_{j}= \begin{cases}\mathbb{R} & \text { if } j=1, \ldots, r_{1}, \\ \mathbb{C} & \text { if } j=r_{1}+1, \ldots, r_{1}+r_{2} .\end{cases}
$$

Let $v(Q)=\left(v^{(1)}, \ldots, v^{\left(r_{1}+r_{2}\right)}\right)$ be the $v$-coordinate of the vector Iwasawa decomposition, and

$$
N(v(Q))=\prod_{j=1}^{r_{1}+r_{2}}\left(v^{(j)}\right)^{N_{j}}
$$

be the norm of $v(Q)$, where

$$
N_{j}= \begin{cases}1 & \text { if } j=1, \ldots, r_{1} \\ 2 & \text { if } j=r_{1}+1, \ldots, r_{1}+r_{2}\end{cases}
$$


Definition 2.4. The Eisenstein series corresponding to the cusp $x_{i}$ is defined by

$$
E_{\left[x_{i}\right]}(Q, s)=N_{K / \mathbb{Q}}\left(\mathfrak{a}_{i}\right)^{2 s} \sum_{\gamma \in \Gamma_{x_{i}} \backslash \mathrm{SL}_{2}\left(\mathcal{O}_{K}\right)} N\left(v\left(Q\left[\gamma A_{i}\right]\right)\right)^{-s}, \quad \operatorname{Re}(s)>1 .
$$

Remark 2.5. We will show in the proof of Proposition 2.7 that $E_{\left[x_{i}\right]}(Q, s)$ depends only on the cusp class $\left[x_{i}\right]$.

We now form the vector Eisenstein series from the Eisenstein series corresponding to the cusp classes.

Definition 2.6. The vector Eisenstein series is defined by

$$
\overrightarrow{\mathbf{E}}(Q, s)=\left(E_{\left[x_{1}\right]}(Q, s), \ldots, E_{\left[x_{h}\right]}(Q, s)\right)^{t} .
$$

In the remaining part of this section we make precise the relationship between the Eisenstein series $\overrightarrow{\mathbf{E}}(Q, s)$ and the Eisenstein series $\overrightarrow{\mathbf{E}}(w, s)$ studied by Efrat and Sarnak in [ES].

Fix an embedding of $K=\mathbb{Q}(\sqrt{-D})$ into $\mathbb{C}$. Then $\Gamma_{D}=\operatorname{PSL}_{2}\left(\mathcal{O}_{D}\right)$ is a discrete subgroup of $\operatorname{Isom}^{+}\left(\mathbb{H}^{3}\right)=\mathrm{PSL}_{2}(\mathbb{C})$ such that $X_{D}=\Gamma_{D} \backslash \mathbb{H}^{3}$ is a non-compact, finite volume arithmetic orbifold. There is an identification of $\mathbb{H}^{3}$ with the quaternionic upper half-plane

$$
\mathbb{H}^{c}=\left\{w=x_{1}+i x_{2}+j y+k t: z=x_{1}+i x_{2} \in \mathbb{C}, y>0, t=0\right\} .
$$

The group $\mathrm{PSL}_{2}(\mathbb{C})$ acts on $\mathbb{H}^{c}$ by linear fractional transformations. Let $x_{1}=$ $\infty, x_{2}, \ldots, x_{h}$ be a complete set of cusps for the fundamental domain $\mathcal{F}$ of $X_{D}$ and let $\Gamma_{x_{i}}$ be the stabilizer of the cusp $x_{i}$. Assume that $D \neq 1,3$, so that $\Gamma_{D}$ is torsion-free. One can choose (not necessarily uniquely) $\sigma_{i} \in \mathrm{PSL}_{2}(\mathbb{C})$ such that $\sigma_{i}\left(x_{i}\right)=\infty$ and

$$
\sigma_{i} \Gamma_{x_{i}} \sigma_{i}^{-1}=\left\{\left(\begin{array}{ll}
1 & l \\
0 & 1
\end{array}\right): l \in L_{i}\right\}
$$

where $L_{i}$ is a lattice in $\mathbb{C}$ whose fundamental domain has volume one.

For $w$ in $\mathbb{H}^{c}$ let

$$
w^{(i)}=\sigma_{i} w=\left(y\left(\sigma_{i} w\right), z\left(\sigma_{i} w\right)\right) .
$$

Then the Eisenstein series associated to the cusp $x_{i}$ is defined by

$$
E_{i}(w, s)=\sum_{\gamma \in \Gamma_{x_{i}} \backslash \Gamma_{D}} y^{(i)}(\gamma w)^{s}, \quad \operatorname{Re}(s)>2,
$$

and the corresponding vector Eisenstein series is defined by

$$
\overrightarrow{\mathbf{E}}(w, s)=\left(E_{1}(w, s), \ldots, E_{h}(w, s)\right)^{t} .
$$

The quaternions $\mathbb{H}$ are the elements of the division ring

$$
\mathbb{H}=\mathbb{R} \oplus \mathbb{R} i \oplus \mathbb{R} j \oplus \mathbb{R} k,
$$

where $i j=k=-j i, j k=i=-k j, k i=j=-i k$, and $i^{2}=j^{2}=k^{2}=-1$. The norm of a quaternion

$$
w=x_{1}+i x_{2}+j y+k t \in \mathbb{H}
$$

is defined by

$$
N(w)=x_{1}^{2}+x_{2}^{2}+y^{2}+t^{2}
$$


Let $\mathfrak{a}_{i}$ be the integral ideal corresponding to the cusp $x_{i}$. Define the Eisenstein series

$$
\widetilde{E}_{i}(w, s)=\alpha_{i}^{s} \sum_{\substack{(c, d) \in \mathfrak{a}_{i} \times \mathfrak{a}_{i} \\(c, d)=\mathfrak{a}_{i}}}\left(\frac{y}{N(c w+d)}\right)^{s}, \quad w \in \mathbb{H}^{3}, \quad \operatorname{Re}(s)>2,
$$

where $\alpha_{i}=N_{K / \mathbb{Q}}\left(\mathfrak{a}_{i}\right) \sqrt{2} / d_{K}^{\frac{1}{4}}$ is a normalizing factor chosen so that $\mathcal{F}$ has volume one. Efrat and Sarnak [ES, Proposition 2.3] proved that $\widetilde{E}_{i}(w, s)=E_{i}(w, s)$.

Proposition 2.7. Let $K$ be imaginary quadratic. Then

$$
2 \beta^{s} E_{\left[x_{i}\right]}\left(Q_{w}, \frac{s}{2}\right)=\widetilde{E}_{i}(w, s), \quad w \in \mathbb{H}^{3}, \quad \operatorname{Re}(s)>2,
$$

where $\beta=\sqrt{2} / d_{K}^{\frac{1}{4}}$, and

$$
Q_{w}:=\left(\begin{array}{cc}
y+\frac{|z|^{2}}{y} & \frac{z}{y} \\
\frac{z}{y} & y^{-1}
\end{array}\right) \in \mathcal{S} \mathcal{Q}_{K}^{2}
$$

(here $w=z+j y$ and $\left.z=x_{1}+i x_{2}\right)$.

Proof. There is a sequence of maps

$$
\mathbb{H}^{3} \rightarrow \mathrm{SL}_{2}(\mathbb{C}) / \mathrm{SU}(2) \rightarrow \mathcal{S} \mathcal{Q}_{K}^{2}
$$

given by

$$
w \mapsto g \mathrm{SU}(2) \mapsto g \bar{g}^{t},
$$

where

$$
g=\left(\begin{array}{cc}
\sqrt{y} & \frac{z}{\sqrt{y}} \\
0 & \sqrt{y}^{-1}
\end{array}\right)
$$

and

$$
Q_{w}:=g \bar{g}^{t}=\left(\begin{array}{cc}
y+\frac{|z|^{2}}{y} & \frac{z}{y} \\
\frac{\bar{z}}{y} & y^{-1}
\end{array}\right) .
$$

By a straightforward calculation one can show that $v\left(Q\left[\gamma A_{i}\right]\right)=Q[a]$, where $a=(c, d)$ is the first column of $\gamma A_{i}$. As $\gamma$ runs over a complete set of representatives of $\Gamma_{x_{i}} \backslash \mathrm{SL}_{2}\left(\mathcal{O}_{K}\right)$, the columns $a$ run over a complete set of generators of $\mathfrak{a}_{i}$ which are non-associate modulo $U_{K}$ (recall that $a$ is associate to $a^{\prime}$ modulo $U_{K}$ if and only if there exists a unit $\epsilon$ such that $a=\epsilon a^{\prime}$ ). It follows that

$$
E_{\left[x_{i}\right]}(Q, s)=N_{K / \mathbb{Q}}\left(\mathfrak{a}_{i}\right)^{2 s} \sum_{\substack{a \in \mathfrak{a}_{i}^{2} / U_{K} \\(c, d)=\mathfrak{a}_{i}}} \prod_{j=1}^{r_{1}+r_{2}} Q^{(j)}\left[a^{(j)}\right]^{-N_{j} s}, \quad \operatorname{Re}(s)>1 .
$$

Note that the argument in the preceding paragraph shows that $E_{\left[x_{i}\right]}(Q, s)$ depends only on the cusp class $\left[x_{i}\right]$.

For $K$ imaginary quadratic, the Eisenstein series can be expressed as

$$
E_{\left[x_{i}\right]}(Q, s)=N_{K / \mathbb{Q}}\left(\mathfrak{a}_{i}\right)^{2 s} \sum_{\substack{a \in \mathfrak{a}_{i}^{2} / U_{K} \\(c, d)=\mathfrak{a}_{i}}} Q[\bar{a}]^{-2 s},
$$

where we have used that the non-trivial embedding of $K$ is complex conjugation. 
Let $Q=Q_{w}$. Then

$$
\begin{aligned}
Q_{w}[\bar{a}] & =(c, d) \cdot\left(\begin{array}{cc}
y+\frac{|z|^{2}}{y} & \frac{z}{y} \\
\frac{\bar{z}}{y} & y^{-1}
\end{array}\right) \cdot\left(\frac{\bar{c}}{d}\right) \\
& =|c|^{2}\left(y+\frac{|z|^{2}}{y}\right)+\bar{c} d \frac{\bar{z}}{y}+c \bar{d} \frac{z}{y}+\frac{|d|^{2}}{y} \\
& =\frac{1}{y}\left(|c|^{2} N(w)+2 \operatorname{Re}(\bar{c} d \bar{z})+|d|^{2}\right) .
\end{aligned}
$$

We claim that

$$
N(c w+d)=|c|^{2} N(w)+2 \operatorname{Re}(\bar{c} d \bar{z})+|d|^{2},
$$

and thus

$$
Q_{w}^{-1}[\bar{a}]=\frac{y}{N(c w+d)} .
$$

Combining these calculations yields

$$
E_{\left[x_{i}\right]}\left(Q_{w}, \frac{s}{2}\right)=\frac{N_{K / \mathbb{Q}}\left(\mathfrak{a}_{i}\right)^{s}}{\left|U_{K}\right|} \sum_{\substack{(c, d) \in \mathfrak{a}_{i} \times \mathfrak{a}_{i} \\(c, d)=\mathfrak{a}_{i}}}\left(\frac{y}{N(c w+d)}\right)^{s} .
$$

Write $N_{K / \mathbb{Q}}\left(\mathfrak{a}_{i}\right)=\alpha_{i} / \beta$, and observe that since $D \neq 1,3, \# U_{K}=2$. Then

$$
E_{\left[x_{i}\right]}\left(Q_{w}, \frac{s}{2}\right)=\frac{\alpha_{i}^{s}}{2 \beta^{s}} \sum_{\substack{(c, d) \in \mathfrak{a}_{i} \times \mathfrak{a}_{i} \\(c, d)=\mathfrak{a}_{i}}}\left(\frac{y}{N(c w+d)}\right)^{s}=\frac{1}{2 \beta^{s}} \tilde{E}_{i}(w, s),
$$

or equivalently,

$$
2 \beta^{s} E_{\left[x_{i}\right]}\left(Q_{w}, \frac{s}{2}\right)=\tilde{E}_{i}(w, s) .
$$

It remains to prove the claim. Write $c=c_{1}+i c_{2}$ and $d=d_{1}+i d_{2}$. Then

$$
\begin{aligned}
c w+d & =\left(c_{1}+i c_{2}\right)\left(x_{1}+i x_{2}+j y\right)+\left(d_{1}+i d_{2}\right) \\
& =\left(c_{1} x_{1}-c_{2} x_{2}+d_{1}\right)+i\left(c_{1} x_{2}+c_{2} x_{1}+d_{2}\right)+j\left(c_{1} y\right)+k\left(c_{2} y\right),
\end{aligned}
$$

where we have used that $i^{2}=-1$ and $i j=k$ in $\mathbb{H}$. Therefore, after expanding, we find that

$$
\begin{aligned}
N(c w+d)= & \left(c_{1} x_{1}-c_{2} x_{2}+d_{1}\right)^{2}+\left(c_{1} x_{2}+c_{2} x_{1}+d_{2}\right)^{2}+\left(c_{1} y\right)^{2}+\left(c_{2} y\right)^{2} \\
= & \left(c_{1}^{2}+c_{2}^{2}\right)\left(x_{1}^{2}+x_{2}^{2}+y^{2}\right)+\left(d_{1}^{2}+d_{2}^{2}\right) \\
& +2\left(c_{1} d_{1} x_{1}+c_{2} d_{2} x_{1}+c_{1} d_{2} x_{2}-c_{2} d_{1} x_{2}\right) \\
= & |c|^{2} N(w)+|d|^{2}+2\left(c_{1} d_{1} x_{1}+c_{2} d_{2} x_{1}+c_{1} d_{2} x_{2}-c_{2} d_{1} x_{2}\right) .
\end{aligned}
$$

Finally,

$\bar{c} d \bar{z}=c_{1} d_{1} x_{1}+c_{2} d_{2} x_{1}+c_{1} d_{2} x_{2}-c_{2} d_{1} x_{2}+i\left(c_{1} d_{2} x_{1}-c_{1} d_{1} x_{2}-c_{2} d_{1} x_{1}-c_{2} d_{2} x_{2}\right)$, so that

$$
\operatorname{Re}(\bar{c} d \bar{z})=c_{1} d_{1} x_{1}+c_{2} d_{2} x_{1}+c_{1} d_{2} x_{2}-c_{2} d_{1} x_{2} .
$$




\section{The VECTOR EPSTEIN ZETA FUnCTION}

Let $\mathcal{R}\left(\mathfrak{a}^{n}\right)$ be a complete set of non-zero, non-associate elements of $\mathfrak{a}^{n}$.

Definition 3.1. The ideal class Epstein zeta function associated to a positive $n$ form $Q$ in $\mathcal{Q}_{K}^{n}$ is defined by

$$
Z_{n}(Q,[\mathfrak{a}], s)=N_{K / \mathbb{Q}}(\mathfrak{a})^{2 s} \sum_{a \in \mathcal{R}\left(\mathfrak{a}^{n}\right)} \prod_{\nu \in S_{\infty}} Q^{(\nu)}\left[a^{(\nu)}\right]^{-N_{\nu} s}, \quad \operatorname{Re}(s)>\frac{n}{2},
$$

where $S_{\infty}$ is a collection of $r_{1}+r_{2}$ infinite places of $K$ and $N_{\nu}=N_{j}$ at the appropriate places $\nu$.

Remark 3.2. The factor $N_{K / \mathbb{Q}}(\mathfrak{a})^{2 s}$ insures that $Z_{n}(Q,[\mathfrak{a}], s)$ depends only on the ideal class $[\mathfrak{a}]$.

Remark 3.3. The zeta function $Z_{n}(Q,[\mathfrak{a}], s)$ has been studied extensively by A. Terras (see $[\mathrm{T}]$ ).

We will establish the following functional equation for $Z_{n}(Q,[\mathfrak{a}], s)$.

Theorem 3.4. Let $A=2^{-r_{2}} d_{K}^{\frac{1}{2}} \pi^{-\frac{N}{2}}$ and

$$
F(Q,[\mathfrak{a}], s)=A^{2 s} \Gamma(s)^{r_{1}} \Gamma(2 s)^{r_{2}} Z_{n}(Q,[\mathfrak{a}], s) .
$$

Then $F(Q,[\mathfrak{a}], s)$ is analytic on $\mathbb{C}$ except for simple poles at $s=0, \frac{n}{2}$, and

$$
F(Q,[\mathfrak{a}], s)=\left\|Q^{-1}\right\|^{\frac{1}{2}} F\left(Q^{-1},\left[\mathfrak{a}^{*}\right], \frac{n}{2}-s\right) .
$$

One forms the vector Epstein zeta function of $Q$ from the ideal class zeta functions.

Definition 3.5. The vector Epstein zeta function of $Q$ is defined by

$$
\overrightarrow{\mathbf{Z}}_{n}(Q, s)=\left(Z_{n}\left(Q,\left[\mathfrak{a}_{1}\right], s\right), \ldots, Z_{n}\left(Q,\left[\mathfrak{a}_{h}\right], s\right)\right)^{t} .
$$

Using Theorem 3.4, we obtain the following functional equation for $\overrightarrow{\mathbf{Z}}_{n}(Q, s)$.

Theorem 3.6. The vector Epstein zeta function $\overrightarrow{\mathbf{Z}}_{n}(Q, s)$ satisfies the functional equation

$$
\overrightarrow{\mathbf{Z}}_{n}(Q, s)=\frac{G\left(2\left(\frac{n}{2}-s\right)\right)}{G(2 s)} \sqrt{\mathbf{D}\left(Q^{-1}\right)} \cdot \mathbf{P} \cdot \overrightarrow{\mathbf{Z}}_{n}\left(Q^{-1}, \frac{n}{2}-s\right) .
$$

\section{A Theta transformation Formula}

We will need the following theta transformation formula in the proof of Theorem 3.4

Proposition 4.1. Let $t^{(1)}, \ldots, t^{(N)}$ be positive real numbers with $t^{\left(r_{1}+r_{2}+j\right)}=$ $t^{\left(r_{1}+j\right)}$ for $j=1, \ldots, r_{2}$. Then

$$
\sum_{a \in \mathfrak{a}^{n}} \exp (-\pi \operatorname{Tr}(Q[a] t))=\frac{1}{\|Q\|^{\frac{1}{2}}\|t\|^{\frac{n}{2}} d_{\mathfrak{a}}^{\frac{n}{2}}} \sum_{a^{\prime} \in\left(\mathfrak{a}^{*}\right)^{n}} \exp \left(-\pi \operatorname{Tr}\left(Q^{-1}\left[a^{\prime}\right] t^{-1}\right)\right),
$$

where

$$
\operatorname{Tr}(Q[a] t)=\sum_{j=1}^{N} Q^{(j)}\left[a^{(j)}\right] t^{(j)}
$$


Proof. Let $K \otimes_{\mathbb{Q}} \mathbb{R}$ be the $N$-dimensional real Minkowski space. Then the function

$$
g(x):=\exp (-\pi \operatorname{Tr}(Q[x] t)), \quad x \in\left(K \otimes_{\mathbb{Q}} \mathbb{R}\right)^{n},
$$

is in the Schwartz class $S\left(\left(K \otimes_{\mathbb{Q}} \mathbb{R}\right)^{n}\right)$.

The ideal $\mathfrak{a}^{n}$ is a lattice of full rank in $\left(K \otimes_{\mathbb{Q}} \mathbb{R}\right)^{n}$. Choose a Haar measure $\mu_{H}$ on $\left(K \otimes_{\mathbb{Q}} \mathbb{R}\right)^{n}$ such that $\mu_{H}\left(\left(K \otimes_{\mathbb{Q}} \mathbb{R}\right)^{n} / \mathfrak{a}^{n}\right)=1$.

From the preceding observations, $g(x)$ is $\left((K \otimes \mathbb{Q} \mathbb{R})^{n}, \mathfrak{a}^{n}\right)$-admissible in the sense of [W], so that by Poisson summation,

$$
\sum_{a \in \mathfrak{a}^{n}} g(a)=\sum_{a^{\prime} \in\left(\mathfrak{a}^{n}\right)^{*}} \hat{g}\left(a^{\prime}\right),
$$

where the Fourier transform is defined by

$$
\hat{g}(z)=\int_{\left(K \mathbb{Q}_{\mathbb{R}}\right)^{n}} g(x) e^{-2 \pi i \operatorname{Tr}\left(z^{T} x\right)} d \mu_{H}(x) .
$$

Let $\left\{\alpha_{1}, \ldots, \alpha_{N}\right\}$ be a basis for $\mathfrak{a}$ over $\mathbb{Z}$. Then $\mathfrak{a}=\bigoplus_{j=1}^{N} \mathbb{Z} \alpha_{j}$ and $K \otimes_{\mathbb{Q}} \mathbb{R}=$ $\bigoplus_{j=1}^{N} \mathbb{R} \alpha_{j}$. It follows that the map $\bigoplus_{j=1}^{N} \mathbb{R} \alpha_{j} \rightarrow \bigoplus_{j=1}^{r_{1}+r_{2}} V_{j}$ defined by

$$
x=\sum_{j=1}^{N} x_{j} \alpha_{j} \mapsto\left(\sum_{j=1}^{N} x_{j} \alpha_{j}^{(1)}, \ldots, \sum_{j=1}^{N} x_{j} \alpha_{j}^{\left(r_{1}+r_{2}\right)}\right)=y
$$

is an isomorphism.

Let $d_{\mathfrak{a}}$ be the absolute value of the discriminant of $\mathfrak{a}$. It can be shown that the absolute value of the determinant of the Jacobian

$$
\begin{array}{r}
\left|\frac{\partial\left(y^{(1)}, \ldots, y^{\left(r_{1}\right)}, \operatorname{Re}\left(y^{\left(r_{1}+1\right)}\right), \operatorname{Im}\left(y^{\left(r_{1}+1\right)}\right), \ldots, \operatorname{Re}\left(y^{\left(r_{1}+r_{2}\right)}\right), \operatorname{Im}\left(y^{\left(r_{1}+r_{2}\right)}\right)\right)}{\partial\left(x_{1}, \ldots, x_{N}\right)}\right| \\
=2^{-r_{2}} d_{\mathfrak{a}}^{\frac{1}{2}} .
\end{array}
$$

Thus we find that under the transformation (4.1), the Haar measure becomes

$$
d \mu_{H}(x)=\left(2^{n}\right)^{r_{2}} d_{\mathfrak{a}}^{-\frac{n}{2}} \prod_{j=1}^{r_{1}+r_{2}} d y^{(j)},
$$

where $d y^{(j)}$ is Lebesque measure on $V_{j}^{n}$. Furthermore, observe that the trace can be expressed as

$$
\operatorname{Tr}(Q[x] t)=\sum_{j=1}^{r_{1}+r_{2}} N_{j} Q^{(j)}\left[x^{(j)}\right] t^{(j)} .
$$

By combining these facts, we find that the Fourier transform can be written as

$$
\begin{aligned}
\hat{g}(z)= & d_{\mathfrak{a}}^{-\frac{n}{2}} \prod_{j=1}^{r_{1}} \int_{\mathbb{R}^{n}} \exp \left(-\pi Q^{(j)}\left[y^{(j)}\right] t^{(j)}\right) e^{-2 \pi i \operatorname{Tr}\left(z^{(j)^{T}} y^{(j)}\right)} d y^{(j)} \\
& \times \prod_{j=r_{1}+1}^{r_{2}} \int_{\mathbb{C}^{n}} \exp \left(-2 \pi Q^{(j)}\left[y^{(j)}\right] t^{(j)}\right) e^{-2 \pi i \operatorname{Tr}\left(z^{(j)^{T}} y^{(j)}\right)} 2^{n} d y^{(j)} .
\end{aligned}
$$


Using standard properties of the Fourier transform and the change of variables formula, we find that

$$
\int_{\mathbb{R}^{n}} \exp \left(-\pi Q^{(j)}\left[y^{(j)}\right] t^{(j)}\right) e^{-2 \pi i \operatorname{Tr}\left(z^{(j)^{T}} y^{(j)}\right)} d y^{(j)}=\frac{\exp \left(-\pi Q^{(j)^{-1}}\left[z^{(j)}\right] t^{(j)^{-1}}\right)}{t^{(j)^{\frac{n}{2}}} \sqrt{\left|\operatorname{det}\left(Q^{(j)}\right)\right|}}
$$

and

$$
\int_{\mathbb{C}^{n}} \exp \left(-2 \pi Q^{(j)}\left[y^{(j)}\right] t^{(j)}\right) e^{-2 \pi i \operatorname{Tr}\left(z^{(j) T} y^{(j)}\right)} 2^{n} d y^{(j)}=\frac{\exp \left(-2 \pi Q^{(j)^{-1}}\left[z^{(j)}\right] t^{(j)^{-1}}\right)}{t^{(j)^{n}}\left|\operatorname{det}\left(Q^{(j)}\right)\right|} .
$$

Substitute these integrals into the formula for $\hat{g}$ and take products to obtain

$$
\hat{g}(z)=\frac{1}{\|Q\|^{\frac{1}{2}}\|t\|^{\frac{n}{2}} d_{\mathfrak{a}}^{\frac{n}{2}}} \exp \left(-\pi \operatorname{Tr}\left(Q^{-1}[z] t^{-1}\right) .\right.
$$

The proposition follows by substituting the formula for $\hat{g}$ into the right-hand side of the Poisson summation formula.

\section{Proof of Theorem 3.4}

Express the absolute value of the discriminant of $\mathfrak{a}$ as $d_{\mathfrak{a}}=N_{K / \mathbb{Q}}(\mathfrak{a})^{2} d_{K}$. By applying the identity

$$
\frac{\Gamma\left(\frac{s}{2}\right)}{a^{s}}=\int_{0}^{\infty} \exp \left(-a^{2} y\right) y^{\frac{s}{2}} \frac{d y}{y}, \quad a>0,
$$

at the real and complex places as in the proof of the functional equation for Hecke $L$-functions, we obtain the Mellin transform

$$
F\left(Q,[\mathfrak{a}], \frac{s}{2}\right)=\int_{0}^{\infty} \cdots \int_{0}^{\infty} \sum_{a \in \mathcal{R}\left(\mathfrak{a}^{n}\right)} \exp \left(-\pi d_{\mathfrak{a}}^{-\frac{1}{N}} \sum_{\nu \in S_{\infty}} N_{\nu} Q^{(\nu)}\left[a^{(\nu)}\right] y_{\nu}\right)\|y\|^{\frac{s}{2}} \frac{d y}{y}
$$

where

$$
\|y\|=\prod_{\nu \in S_{\infty}} y_{\nu}^{N_{\nu}} \quad \text { and } \quad \frac{d y}{y}=\prod_{\nu \in S_{\infty}} \frac{d y_{\nu}}{y_{\nu}} .
$$

Define the multiplicative group $G=\prod_{\nu \in S_{\infty}}\left(\mathbb{R}_{\nu}^{+}\right)^{*}$, and the map $|\cdot|: K^{*} \rightarrow G$ by $|\alpha|=\left(\left|\alpha^{(\nu)}\right|\right)_{\nu \in S_{\infty}}$. Observe that ker $|\cdot|$ is the group of roots of unity and that $V=\left|U_{K}\right|$ is a discrete subgroup of the norm one hypersurface $G^{0}$ defined by $G^{0}=\{y \in G:\|y\|=1\}<G$, with compact quotient $G^{0} / V$.

For $y \in G$, write $y=t^{\frac{1}{N}} c$ uniquely by setting $t=\|y\|$ and $c=\frac{y}{\|y\|^{\frac{1}{N}}}$. We thus obtain the decomposition $G=G^{0} \times\left(\mathbb{R}^{+}\right)^{*}$.

Let $d^{*} c$ be the unique Haar measure on the multiplicative group $G^{0}$ such that the canonical Haar measure $d y / y$ on $G$ becomes $\frac{d y}{y}=d^{*} c \times \frac{d t}{t}$. We can now express the Mellin transform as

$$
F\left(Q,[\mathfrak{a}], \frac{s}{2}\right)=\int_{0}^{\infty} \int_{G^{0}} \sum_{a \in \mathcal{R}\left(\mathfrak{a}^{n}\right)} \exp \left(-\pi t^{\frac{1}{N}} d_{\mathfrak{a}}^{-\frac{1}{N}} \sum_{\nu \in S_{\infty}} N_{\nu} Q^{(\nu)}\left[a^{(\nu)}\right] c_{\nu}\right) d^{*} c t^{\frac{s}{2}} \frac{d t}{t} .
$$


Choose a fundamental domain $E$ for the action of the group $\left|U_{K}\right|^{2}=\left\{|\epsilon|^{2}: \epsilon \epsilon\right.$ $\left.U_{K}\right\}$ on $G^{0}$ which decomposes the norm one hypersurface into the disjoint union

$$
G^{0}=\bigcup_{\eta \in\left|U_{K}\right|} \eta^{2} E
$$

Then

$$
\begin{aligned}
& \int_{G^{0}} \sum_{a \in \mathcal{R}\left(\mathfrak{a}^{n}\right)} \exp \left(-\pi t^{\frac{1}{N}} d_{\mathfrak{a}}^{-\frac{1}{N}} \sum_{\nu \in S_{\infty}} N_{\nu} Q^{(\nu)}\left[a^{(\nu)}\right] c_{\nu}\right) d^{*} c \\
& =\int_{E} \frac{1}{w_{K}} \sum_{a \in \mathfrak{a}^{n} \backslash\{0\}} \exp \left(-\pi t^{\frac{1}{N}} d_{\mathfrak{a}}^{-\frac{1}{N}} \sum_{\nu \in S_{\infty}} N_{\nu} Q^{(\nu)}\left[a^{(\nu)}\right] c_{\nu}\right) d^{*} c
\end{aligned}
$$

where $w_{K}$ is the number of roots of unity in $K$.

Substitute (5.1) in the Mellin transform to obtain

$$
F\left(Q,[\mathfrak{a}], \frac{s}{2}\right)=\int_{0}^{\infty} \int_{E} \frac{1}{w_{K}}\left[\Theta_{n}\left(Q, \mathfrak{a}, t^{\frac{1}{N}} d_{\mathfrak{a}}^{-\frac{1}{N}} c\right)-1\right] d^{*} c t^{\frac{s}{2}} \frac{d t}{t},
$$

where

$$
\Theta_{n}\left(Q, \mathfrak{a}, t^{\frac{1}{N}} d_{\mathfrak{a}}^{-\frac{1}{N}} c\right)=\sum_{a \in \mathfrak{a}^{n}} \exp \left(-\pi t^{\frac{1}{N}} d_{\mathfrak{a}}^{-\frac{1}{N}} \sum_{\nu \in S_{\infty}} N_{\nu} Q^{(\nu)}\left[a^{(\nu)}\right] c_{\nu}\right) .
$$

Now, a simple integration allows us to express the Mellin transform as

$$
\begin{aligned}
F\left(Q,[\mathfrak{a}], \frac{s}{2}\right)= & \int_{0}^{1} \int_{E} \frac{1}{w_{K}} \Theta_{n}\left(Q, \mathfrak{a}, t^{\frac{1}{N}} d_{\mathfrak{a}}^{-\frac{1}{N}} c\right) d^{*} c t^{\frac{s}{2}} \frac{d t}{t}-\frac{2 \mu^{*}(E)}{w_{K} s} \\
& +\int_{1}^{\infty} \int_{E} \frac{1}{w_{K}}\left[\Theta_{n}\left(Q, \mathfrak{a}, t^{\frac{1}{N}} d_{\mathfrak{a}}^{-\frac{1}{N}} c\right)-1\right] d^{*} c t^{\frac{s}{2}} \frac{d t}{t}
\end{aligned}
$$

where $\mu^{*}(E)$ is the measure of the pullback of $E$.

Let $t=\tau^{-1}$ and $d t=-d \tau / \tau^{2}$ in the first integral on the right-hand side of (5.2). Using the invariance of $d^{*} c$ under the transformation $c \mapsto c^{-1}$, the functional equation for the theta function $\Theta_{n}(Q, \mathfrak{a}, z)$ given in Proposition [5.1, and another simple integration, we find upon making the substitution $s \rightarrow 2 s$ that

$$
\begin{aligned}
& F(Q,[\mathfrak{a}], s)=\int_{1}^{\infty} \int_{E} \frac{1}{w_{K}}\left[\Theta_{n}\left(Q, \mathfrak{a}, t^{\frac{1}{N}} d_{\mathfrak{a}}^{-\frac{1}{N}} c\right)-1\right] d^{*} c t^{s} \frac{d t}{t}-\frac{\mu^{*}(E)}{w_{K} s} \\
& \quad+\int_{1}^{\infty} \int_{E} \frac{\left\|Q^{-1}\right\|^{\frac{1}{2}}}{w_{K}}\left[\Theta_{n}\left(Q^{-1}, \mathfrak{a}^{*}, t^{\frac{1}{N}} d_{\mathfrak{a}^{*}}^{-\frac{1}{N}} c\right)-1\right] d^{*} c t^{\left(\frac{n}{2}-s\right)} \frac{d t}{t}-\frac{\mu^{*}(E)\left\|Q^{-1}\right\|^{\frac{1}{2}}}{w_{K}\left(\frac{n}{2}-s\right)} .
\end{aligned}
$$

From (5.3), it is clear that $F(Q,[\mathfrak{a}], s)$ is analytic on $\mathbb{C}$ except for simple poles at $s=0, \frac{n}{2}$. Make the substitutions $s \rightarrow \frac{n}{2}-s, \mathfrak{a} \rightarrow \mathfrak{a}^{*}$, and $Q \rightarrow Q^{-1}$ in (5.3) to complete the proof.

In the following proposition, we establish the functional equation for the theta function $\Theta_{n}(Q, \mathfrak{a}, z)$. 
Proposition 5.1. With notation as above we have

$$
\Theta_{n}\left(Q, \mathfrak{a}, t^{\frac{1}{N}} d_{\mathfrak{a}}^{-\frac{1}{N}} c\right)=\frac{1}{t^{\frac{n}{2}}\|Q\|^{\frac{1}{2}}} \Theta_{n}\left(Q^{-1}, \mathfrak{a}^{*}, t^{-\frac{1}{N}} d_{\mathfrak{a}^{*}}^{-\frac{1}{N}} c^{-1}\right) .
$$

Proof. This follows from a straightforward calculation using Proposition 4.1

\section{Proof of Theorem 1.1}

We will need the following proposition (see also $[\mathrm{T}]$ ).

Proposition 6.1. With notation as above we have

$$
\overrightarrow{\mathbf{Z}}_{2}(Q, s)=\left(\zeta_{\left[\mathfrak{a}_{i}^{-1} \mathfrak{a}_{j}\right]}(2 s)\right) \cdot \overrightarrow{\mathbf{E}}(Q, s) .
$$

Proof. Recall from the proof of Proposition 2.7 that

$$
E_{\left[x_{i}\right]}(Q, s)=N_{K / \mathbb{Q}}\left(\mathfrak{a}_{i}\right)^{2 s} \sum_{\substack{a \in \mathcal{R}\left(\mathfrak{a}_{i}^{2}\right) \\\left(a_{1}, a_{2}\right)=\mathfrak{a}_{i}}} \prod_{\nu \in S_{\infty}} Q^{(\nu)}\left[a^{(\nu)}\right]^{-N_{\nu} s}, \quad \operatorname{Re}(s)>1 .
$$

Therefore,

$$
\begin{aligned}
& Z_{2}\left(Q,\left[\mathfrak{a}_{i}\right], s\right)=N_{K / \mathbb{Q}}\left(\mathfrak{a}_{i}\right)^{2 s} \sum_{a \in \mathcal{R}\left(\mathfrak{a}_{i}^{2}\right)} \prod_{\nu \in S_{\infty}} Q^{(\nu)}\left[a^{(\nu)}\right]^{-N_{\nu} s} \\
& =N_{K / \mathbb{Q}}\left(\mathfrak{a}_{i}\right)^{2 s} \sum_{\substack{\mathfrak{a}_{i} \mid \mathfrak{b} \\
\sum_{\left(\in \in \mathcal{R}\left(\mathfrak{b}^{2}\right)\right.}}} \prod_{\nu \in S_{\infty}} Q^{(\nu)}\left[a^{(\nu)}\right]^{-N_{\nu} s} \\
& =\sum_{\mathfrak{a}_{i} \mid \mathfrak{b}} N_{K / \mathbb{Q}}\left(\mathfrak{a}_{i}^{-1} \mathfrak{b}\right)^{-2 s} N_{K / \mathbb{Q}}(\mathfrak{b})^{2 s} \sum_{\substack{\left.a \in \mathcal{R}\left(\mathfrak{b}^{2}\right)\right) \\
\left(a_{1}, a_{2}\right)=\mathfrak{b}}} \prod_{\nu \in S_{\infty}} Q^{(\nu)}\left[a^{(\nu)}\right]^{-N_{\nu} s} .
\end{aligned}
$$

To complete the proof, let $\mathfrak{c}=\mathfrak{a}_{i}^{-1} \mathfrak{b}$ and observe that $\mathfrak{c}$ runs through all integral ideals in the class $\left[\mathfrak{a}_{i}^{-1} \mathfrak{a}_{j}\right]$.

We will also need the determinant of the matrix of ideal class zeta functions. Let $\chi \in \overline{c l\left(\mathcal{O}_{K}\right)}:=\operatorname{Hom}\left(\operatorname{cl}\left(\mathcal{O}_{K}\right), \mathbb{T}\right)$ be a character of the ideal class group. Define the $h \times h$ matrix $\mathbf{M}=\left(\bar{\chi}_{i}\left(\mathfrak{a}_{j}\right)\right)$ whose $(i, j)$-th component $\bar{\chi}_{i}\left(\mathfrak{a}_{j}\right)$ is the complex conjugate of the character $\chi_{i}$ evaluated at the ideal class representative $\mathfrak{a}_{j}$. Further, define the class group $L$-function

$$
L(\chi, s)=\sum_{(0) \neq \mathfrak{a} \subset \mathcal{O}_{K}} \chi(\mathfrak{a}) N_{K / \mathbb{Q}}(\mathfrak{a})^{-s}, \quad \operatorname{Re}(s)>1,
$$

and the $h \times h$ diagonal matrix of $L$-functions

$$
\mathbf{L}(s)=\left(\begin{array}{ccc}
L\left(\chi_{1}, s\right) & & \\
& \ddots & \\
& & L\left(\chi_{h}, s\right)
\end{array}\right) .
$$

Proposition 6.2. With notation as above we have

$$
\left(\zeta_{\left[\mathfrak{a}_{i}^{-1} \mathfrak{a}_{j}\right]}(s)\right)=\mathbf{M}^{-1} \cdot \mathbf{L}(s) \cdot \mathbf{M} .
$$


Proof. Let $\chi \in \widehat{c l\left(\mathcal{O}_{K}\right)}$. Then

$$
\begin{aligned}
\sum_{i=1}^{h} \bar{\chi}\left(\mathfrak{a}_{i}\right) \zeta_{\left[\mathfrak{a}_{i}^{-1} \mathfrak{a}_{j}\right]}(s) & =\sum_{i=1}^{h} \sum_{\mathfrak{b} \in\left[\mathfrak{a}_{i}^{-1} \mathfrak{a}_{j}\right]} \bar{\chi}\left(\mathfrak{a}_{i}\right) N_{K / \mathbb{Q}}(\mathfrak{b})^{-s} \\
& =\bar{\chi}\left(\mathfrak{a}_{j}\right) \sum_{i=1}^{h} \sum_{\mathfrak{b} \in\left[\mathfrak{a}_{i}^{-1} \mathfrak{a}_{j}\right]} \bar{\chi}\left(\mathfrak{b}^{-1}\right) N_{K / \mathbb{Q}}(\mathfrak{b})^{-s} \\
& =\bar{\chi}\left(\mathfrak{a}_{j}\right) \sum_{i=1}^{h} \sum_{\mathfrak{b} \in\left[\mathfrak{a}_{i}^{-1} \mathfrak{a}_{j}\right]} \chi(\mathfrak{b}) N_{K / \mathbb{Q}}(\mathfrak{b})^{-s} \\
& =\bar{\chi}\left(\mathfrak{a}_{j}\right) L(\chi, s) .
\end{aligned}
$$

From Proposition 6.2 and class field theory (see [N]),

$$
\operatorname{det}\left(\zeta_{\left[\mathfrak{a}_{i}^{-1} \mathfrak{a}_{j}\right]}(s)\right)=\prod_{\chi \in c l\left(\mathcal{O}_{K}\right)} L(\chi, s)=\zeta_{H}(s) .
$$

Proof of Theorem 1.1. Let $n=2$ in Theorem 3.6 and apply Proposition 6.1 to both sides of the functional equation (3.1) to obtain

$$
\begin{aligned}
& \left(\zeta_{\left[\mathfrak{a}_{i}^{-1} \mathfrak{a}_{j}\right]}(2 s)\right) \cdot \overrightarrow{\mathbf{E}}(Q, s) \\
& =\frac{G(2(1-s))}{G(2 s)} \sqrt{\mathbf{D}\left(Q^{-1}\right)} \cdot \mathbf{P} \cdot\left(\zeta_{\left[\mathfrak{a}_{i}^{-1} \mathfrak{a}_{j}\right]}(2(1-s))\right) \cdot \overrightarrow{\mathbf{E}}\left(Q^{-1}, 1-s\right) .
\end{aligned}
$$

Because $\zeta_{H}(2 s) \neq 0$ for $\operatorname{Re}(s)>\frac{1}{2}$, (6.1) implies that the matrix $\left(\zeta_{\left[\mathfrak{a}_{i}^{-1} \mathfrak{a}_{j}\right]}(2 s)\right)$ is invertible for $\operatorname{Re}(s)>\frac{1}{2}$. It follows that

$$
\begin{aligned}
& \overrightarrow{\mathbf{E}}(Q, s)= \\
& \frac{G(2(1-s))}{G(2 s)} \sqrt{\mathbf{D}\left(Q^{-1}\right)} \cdot\left(\zeta_{\left[\mathfrak{a}_{i}^{-1} \mathfrak{a}_{j}\right]}(2 s)\right)^{-1} \cdot \mathbf{P} \cdot\left(\zeta_{\left[\mathfrak{a}_{i}^{-1} \mathfrak{a}_{j}\right]}(2(1-s))\right) \cdot \overrightarrow{\mathbf{E}}\left(Q^{-1}, 1-s\right)
\end{aligned}
$$

for $\operatorname{Re}(s)>\frac{1}{2}$. The Eisenstein series $\overrightarrow{\mathbf{E}}(Q, s)$ has a meromorphic continuation to $\mathbb{C}$ (see [La]). Theorem 1.1 follows by uniqueness of analytic continuation.

\section{The Determinant of $\mathbf{P}$}

Proposition 7.1. Let $m$ be the order of the group $\operatorname{cl}\left(\mathcal{O}_{K}\right)[2]$ of 2-torsion in $\operatorname{cl}\left(\mathcal{O}_{K}\right)$. Then

$$
\operatorname{det}(\mathbf{P})=(-1)^{\frac{h-m}{2}} .
$$

Proof. Suppose that $\sigma$ is a permutation of $\operatorname{cl}\left(\mathcal{O}_{K}\right)$ with corresponding permutation matrix $\mathbf{P}_{\sigma}$. Then $\operatorname{det}\left(\mathbf{P}_{\sigma}\right)=(-1)^{l}$, where $l$ is the number of transpositions in the decomposition of $\sigma$ as a product of transpositions. In terms of $\mathbf{P}_{\sigma}, l=\frac{h-|\operatorname{Fix}(\sigma)|}{2}$, where we have divided by two since one transposition corresponds to an exchange of two rows. Specializing to $\sigma^{*}: \mathfrak{a} \rightarrow \mathfrak{a}^{*}$, we see that it suffices to show that $\left|\operatorname{Fix}\left(\sigma^{*}\right)\right|=\left|c l\left(\mathcal{O}_{K}\right)[2]\right|$.

It is a deep theorem of Hecke that the different $\mathfrak{D}_{K}$ is a square in $\operatorname{cl}\left(\mathcal{O}_{K}\right)$ (see [H, Theorem 176]). Therefore, because $\mathfrak{a}^{*}=\left(\mathfrak{a} \mathfrak{D}_{K}\right)^{-1}$, we need only consider the 
permutation $\sigma_{g}: x \rightarrow x^{-1} g^{2}$, where $g$ is a fixed element of $\operatorname{cl}\left(\mathcal{O}_{K}\right)$. But an element $x$ in $\operatorname{cl}\left(\mathcal{O}_{K}\right)$ is fixed by $\sigma_{g}$ if and only if $x^{2}=g^{2}$ if and only if $x=a g$, where $a^{2}=1$. We conclude that $\left|\operatorname{Fix}\left(\sigma_{g}\right)\right|=\left|\operatorname{cl}\left(\mathcal{O}_{K}\right)[2]\right|$.

\section{Proof of Theorem 1.3}

Given the formula for $\Phi(s)$ in Theorem [1.1, it suffices to compute the determinants of the matrices $\mathbf{P}, \sqrt{\mathbf{D}\left(Q^{-1}\right)}$, and $\left(\zeta_{\left[\mathfrak{a}_{i}^{-1} \mathfrak{a}_{j}\right]}(s)\right)$. We have computed the first and third of these in Proposition 7.1 and equation (6.1), respectively, and a straightforward calculation yields $\operatorname{det}\left(\sqrt{\mathbf{D}\left(Q^{-1}\right)}\right)=\left\|Q^{-1}\right\|^{\frac{h}{2}}$.

\section{Proof of Theorem 1.6}

We will need the following lemma, which follows from a calculation using the orthogonality relations for the group characters $\chi \in c \widehat{c\left(\mathcal{O}_{K}\right)}$.

Lemma 9.1. With notation as above we have

$$
\mathbf{M}^{-1} \cdot\left(\begin{array}{cccc}
-1 & & & \\
& 1 & & \\
& & \ddots & \\
& & & 1
\end{array}\right) \cdot \mathbf{M}=-\frac{2}{h}\left(\begin{array}{cccc}
1 & 1 & \cdots & 1 \\
1 & 1 & \cdots & 1 \\
\vdots & \vdots & & \vdots \\
1 & 1 & \cdots & 1
\end{array}\right)+\mathbf{I}_{h} .
$$

Proof of Theorem 1.6. From Theorem 1.1 and Proposition 6.2 we find that

$$
\begin{aligned}
\operatorname{Tr}(\Phi(s)) & =\operatorname{Tr}\left(\frac{G(2(1-s))}{G(2 s)} \sqrt{\mathbf{D}\left(Q^{-1}\right)} \cdot \mathbf{P} \cdot\left(\zeta_{\left[\mathfrak{a}_{i}^{-1} \mathfrak{a}_{j}\right]}(2(1-s))\right) \cdot\left(\zeta_{\left[\mathfrak{a}_{i}^{-1} \mathfrak{a}_{j}\right]}(2 s)\right)^{-1}\right) \\
& =\operatorname{Tr}\left(\frac{G(2(1-s))}{G(2 s)} \sqrt{\mathbf{D}\left(Q^{-1}\right)} \cdot \mathbf{P} \cdot \mathbf{M}^{-1} \cdot \mathbf{L}(2(1-s)) \cdot \mathbf{L}(2 s)^{-1} \cdot \mathbf{M}\right) .
\end{aligned}
$$

Using the Laurent expansion of the Dedekind zeta function $\zeta_{K}(s)$ at $s=1$, it is easily seen that

$$
\lim _{s \rightarrow \frac{1}{2}} \frac{L\left(\chi_{1}, 2(1-s)\right)}{L\left(\chi_{1}, 2 s\right)}=-1 .
$$

Also, observe that $G(s)$ is holomorphic at $s=1$, and for $\chi \neq 1, L(\chi, s)$ is holomorphic at $s=1$. Combining these facts with Lemma 9.1 yields

$$
\begin{aligned}
& \operatorname{Tr}\left(\Phi\left(\frac{1}{2}\right)\right)=\lim _{s \rightarrow \frac{1}{2}} \operatorname{Tr}(\Phi(s)) \\
& =\left\|Q^{-1}\right\|^{\frac{1}{2}} \operatorname{Tr}\left(\mathbf{P} \cdot \mathbf{M}^{-1} \cdot\left(\begin{array}{llll}
-1 & & & \\
& 1 & & \\
& & \ddots & \\
& & & 1
\end{array}\right) \cdot \mathbf{M}\right) \\
& =\left\|Q^{-1}\right\|^{\frac{1}{2}} \operatorname{Tr}\left(\mathbf{P} \cdot \frac{-2}{h}\left(\begin{array}{cccc}
1 & 1 & \cdots & 1 \\
1 & 1 & \cdots & 1 \\
\vdots & \vdots & & \vdots \\
1 & 1 & \cdots & 1
\end{array}\right)+\mathbf{P} \cdot \mathbf{I}_{h}\right) \\
& =\left\|Q^{-1}\right\|^{\frac{1}{2}}(\operatorname{Tr}(\mathbf{P})-2) \text {. }
\end{aligned}
$$


Finally, because the trace of a permutation matrix is the number of fixed points, $\operatorname{Tr}(\mathbf{P})=\left|\operatorname{Fix}\left(\sigma^{*}\right)\right|=m$ by Proposition 7.1 .

\section{REFERENCES}

[ES] I. Efrat and P. Sarnak, The determinant of the Eisenstein matrix and Hilbert class fields, Trans. Amer. Math. Soc. 290 (1985), 815-824. MR792829 (87b:11039)

[H] E. Hecke, Lectures on the Theory of Algebraic Numbers, Springer-Verlag, New York-Berlin, 1981. MR638719 (83m:12001)

[La] R. Langlands, On the functional equations satisfied by Eisenstein series, Lecture Notes in Mathematics, Vol. 544, Springer-Verlag, Berlin-New York, 1976. MR0579181 (58:28319)

[LP] P. Lax and R. Phillips, Scattering theory for automorphic functions, Annals of Mathematics Studies, No. 87, Princeton Univ. Press, Princeton, N.J., 1976. MR0562288 (58:27768)

[N] J. Neukrich, Algebraic Number Theory, Springer-Verlag, Berlin-Heidelberg, 1999. MR1697859 (2000m:11104)

[PS] R. Phillips and P. Sarnak, Perturbation theory for the Laplacian on automorphic functions, J. Amer. Math. Soc. 5 (1992), 1-32. MR.1127079 (92g:11056)

[R] A. Reznikov, Eisenstein matrix and existence of cusp forms in rank one symmetric spaces, Geom. Funct. Anal. 3 (1993), 79-105. MR1204788 (94d:11034)

[Sa] P. Sarnak, On cusp forms. II, Festschrift in honor of I. I. Piatetski-Shapiro on the occasion of his sixtieth birthday, Part II (Ramat Aviv, 1989), 237-250, Israel Math. Conf. Proc., 3, Weizmann, Jerusalem, 1990. MR1159118 (93e:11068)

[Si] C. L. Siegel, Lectures on Advanced Analytic Number Theory, Tata Institute of Fundamental Research, Bombay, 1965. MR0262150 (41:6760)

[So] C. Sorensen, Fourier expansion of Eisenstein series on the Hilbert modular group and Hilbert class fields, Trans. Amer. Math. Soc. 354 (2002), 4847-4869. MR.1926839 (2003m:11069)

[T] A. Terras, Harmonic analysis and symmetric spaces and applications. II, Springer-Verlag, Berlin, 1988. MR955271 (89k:22017)

[W] A. Weil, Basic Number Theory, Springer-Verlag, New York, 1967. MR0234930 (38:3244)

Department of Mathematics, University of Wisconsin, Madison, Wisconsin 53706

E-mail address: masri@math.wisc.edu 